\title{
Outcomes of individuals with profound and partial biotinidase deficiency ascertained by newborn screening in Michigan over 25 years
}

\author{
Allison M. Jay, MD¹, Robert L. Conway, MD¹, Gerald L. Feldman, MD, PhD ${ }^{1-3}$, Fatimah Nahhas, $\mathrm{PhD}^{2,3}$, \\ Linda Spencer, RN, MSN ${ }^{1}$ and Barry Wolf, MD, PhD ${ }^{2,4}$
}

\begin{abstract}
Purpose: Biotinidase deficiency, if untreated, usually results in neurological and cutaneous symptoms. Biotin supplementation markedly improves and likely prevents symptoms in those treated early. All states in the United States and many countries perform newborn screening for biotinidase deficiency. However, there are few studies about the outcomes of the individuals identified by newborn screening.
\end{abstract}

Methods: We report the outcomes of 142 children with biotinidase deficiency identified by newborn screening in Michigan over a 25-year period and followed in our clinic; 22 had profound deficiency and 120 had partial deficiency.

Results: Individuals with profound biotinidase and partial deficiency identified by newborn screening were started on biotin ther- apy soon after birth. With good compliance, these children appeared to have normal physical and cognitive development. Although some children exhibited mild clinical problems, these are unlikely attributable to the disorder. Biotin therapy appears to prevent the development of neurological and cutaneous problems in our population.

Conclusion: Individuals with biotinidase deficiency ascertained by newborn screening and treated since birth appeared to exhibit normal physical and cognitive development. If an individual does develop symptoms, after compliance and dosage issues are excluded, then other causes must be considered.

Genet Med advance online publication 21 August 2014

Key Words: biotin; biotinidase deficiency; inherited metabolic disease; newborn screening; outcomes

\section{INTRODUCTION}

Biotinidase is the enzyme that recycles the water-soluble vitamin biotin, making the coenzyme available for activation of the four biotin-dependent enzymes in humans. ${ }^{1,2}$ Biotinidase deficiency (OMIM 253260) is an autosomal recessively inherited disorder. ${ }^{3}$ Individuals with profound biotinidase deficiency have less than $10 \%$ of mean normal serum biotinidase activity, whereas those with partial biotinidase deficiency have between $10 \%$ and $30 \%$ mean normal serum biotinidase activity. ${ }^{4}$ The clinical features of symptomatic children with profound biotinidase deficiency include neurological features, including hypotonia, lethargy, seizures, ataxia, optic atrophy, hearing loss, and cognitive deficits, which if untreated can progress to coma or death. ${ }^{4,5}$ The cutaneous features include eczematoid skin rash, conjunctivitis, and alopecia. Biotin treatment can markedly resolve many of these features, but if optic atrophy, hearing loss, or cognitive deficits develop, these are usually irreversible. ${ }^{4}$ Those individuals with partial biotinidase deficiency, if untreated, may develop milder symptoms, especially if stressed by an infection. ${ }^{4}$ Biotin therapy appears to prevent the development of symptoms in those started on biotin before symptoms occur. Therefore, all the states in the United States and many countries have added biotinidase deficiency to their newborn screening programs. ${ }^{6,7}$

The first newborn screening for biotinidase deficiency was initiated in Virginia in $1984 .{ }^{8}$ The state of Michigan began screening in 1988. However, even though many states have been screening for a relatively long period of time, few studies have been reported that have examined the outcomes of the individuals identified by newborn screening. In this study, we report the experience and outcomes of children identified with profound and partial biotinidase deficiency in Michigan over a 25-year period.

\section{MATERIALS AND METHODS}

This study is based on a retrospective review of 142 individuals with profound and partial biotinidase deficiency identified by newborn screening in Michigan and several children identified in other states and who had follow-up at the Children's Hospital of Michigan, over 25 years, from 1988 through the end of 2012. We reviewed their retrospective data at their last clinic visit to determine if they have ever had developmental deficits, neurological, dermatological, ophthalmological, or audiological abnormalities, or other health problems. We also ascertained

${ }^{1}$ Department of Pediatrics, Wayne State University School of Medicine, Detroit, Michigan, USA; ${ }^{2}$ Center for Molecular Medicine and Genetics, Wayne State University, Detroit, Michigan, USA; ${ }^{3}$ Department of Pathology, Wayne State University School of Medicine, Detroit, Michigan, USA; ${ }^{4}$ Genetics Research Laboratory, Department of Research Administration, Henry Ford Hospital, Detroit, Michigan, USA. Correspondence: Barry Wolf (barrywolf1@comcast.net) 
their dosage of biotin and reported compliance history for taking biotin.

In our screening program, children are routinely examined in our clinic as soon as possible after diagnosis, at 6 months of age, at 1 year of age, and then annually. We then routinely recommended audiological and ophthalmological evaluations for all newly diagnosed newborns within the first year of life, and then yearly for those with profound biotinidase deficiency and biannually for those with partial biotinidase deficiency. The state of Michigan pays for the biotin supplements for individuals with profound and partial biotinidase deficiency.

\section{Enzymatic analysis}

Confirmational biotinidase activities using the colorimetric assay ${ }^{9}$ were determined at various laboratories, including the Children's Hospital of Michigan at Detroit Medical Center, the Medical College of Virginia at Virginia Commonwealth University, Associated Regional University Pathologists, Mayo Clinic, University of Michigan, and the University of Maryland. All individuals with less than $10 \%$ of mean normal serum biotinidase activity were considered as having profound biotinidase deficiency, ${ }^{4}$ whereas all individuals with between $10 \%$ and $30 \%$ of mean normal biotinidase activity were considered as having partial biotinidase deficiency.

\section{Mutation analysis}

DNA was isolated from blood and sequencing was performed on the entire complementary DNA sequence and exon/intron junction regions of the biotinidase (BTD) gene at the Detroit Medical Center Laboratory or the DNA Diagnostic Laboratory at Henry Ford Hospital. ${ }^{10}$ Targeted mutation panel testing was performed by PerkinElmer Genetics. Mutation results were correlated with the enzymatic results. In several instances, the assignment of a child as having partial biotinidase deficiency or being within the heterozygous profoundly deficient range was resolved through mutation analysis. Novel mutations were confirmed by parental enzymatic activities and mutational analyses. These novel mutations are reported elsewhere. ${ }^{11}$

\section{RESULTS}

Between 1988 and the end of 2012, 32 newborns were confirmed to have profound biotinidase deficiency and 197 newborns were confirmed to have partial biotinidase deficiency. The incidence of combined profound and partial biotinidase deficiency in Michigan was 1 in 14,539, which was considerably higher than had been reported for the worldwide incidence of 1 in 61,000 for both profound and partial biotinidase deficiency. ${ }^{12}$ The incidence of profound biotinidase deficiency was 1 in 101,779 newborns, and the incidence of partial biotinidase deficiency was 1 in 16,533 newborns.

\section{Individuals with profound biotinidase deficiency}

Twenty-two of the 32 children with profound biotinidase deficiency were followed in our clinic. Thirteen of these 20 children had complete and/or targeted mutation analyses (Table
Table 1 Mutation analysis of individuals with profound biotinidase deficiency

\begin{tabular}{ll} 
Mutation 1 & \multicolumn{1}{c}{ Mutation $\mathbf{2}$} \\
\hline c.33fs & p.A171T, p.D444H \\
C.33fs & p.P253S \\
c.33fs & $\begin{array}{l}\text { Targeted sequencing; second } \\
\text { mutation not examined }\end{array}$ \\
\hline p.F149L & p.A171T, p.D444H \\
p.A162V & p.Q456H \\
p.T234I & p.T234I \\
p.C245Y & p.T532M \\
p.P497S & p.P497S \\
p.Q456H & p.A171T, p.D444H \\
p.Q456H & p.V199M \\
p.Q456H & p.R209H \\
p.Q456H & p.T532M \\
p.Q456H & Targeted sequencing; second \\
& mutation not examined \\
\hline
\end{tabular}

1). The ages of these children ranged from 1 to 20 years, with a mean age of 8.3 years. Five cases were confirmed immediately after birth because they had affected siblings. With the exception of a case that was confirmed as being outside of the newborn period in a single child, all of the children were started on biotin at 1 week to 3 weeks of age.

All of these children have been treated with between $10 \mathrm{mg}$ and $15 \mathrm{mg}$ of biotin per day. All of the families of these children indicated good compliance in using the biotin.

None of these children exhibited hypotonia, seizures, developmental delay, optic atrophy, or hearing problems. Seven children had cutaneous issues that included rashes, eczema, and contact dermatitis. Because of the symptoms, biotin was usually increased from $10 \mathrm{mg}$ to $15 \mathrm{mg}$ daily. However, the reports are incomplete or absent regarding the response to the biotin.

Five of these children have had some vision issues, which included astigmatism or requiring glasses or contacts. None of the families indicated problems with compliance.

\section{Individuals with partial biotinidase deficiency}

Of the 197 individuals with partial biotinidase deficiency, 120 were followed in our clinic. The age of the children with partial biotinidase deficiency ranged from 2 weeks old (at their first evaluation visit) to 29 years; the mean age of these individuals was 5.8 years. The median dose of biotin used to treat the individuals with partial biotinidase deficiency was $5 \mathrm{mg}$ per day. Although several parents reported missing occasional doses of biotin (less than 1 week), only five families reported missing the biotin for more than 1 week; none of these five children had symptoms during this period of noncompliance. Therefore, compliance in administering the biotin was also good for families with children with partial deficiency.

Ninety-eight of the 120 individuals had mutation analysis (Table 2). Of these, $95 \%$ or $97 \%$ had at least one D444H mutation. Based on their equivocal enzymatic activity and mutation analysis, three individuals initially thought to have partial biotinidase deficiency were subsequently shown to either have 
Table 2 Mutation analysis of individuals with partial biotinidase deficiency

\begin{tabular}{|c|c|c|}
\hline Mutation 1 & Mutation 2 & Number \\
\hline p.D444H & p.D444H, p.A171T & 27 \\
\hline p.D444H & p.Q456H & 16 \\
\hline p.D444H & c.33fs & 10 \\
\hline p.D444H & p.D252G & 5 \\
\hline p.D444H & p.R538C & 5 \\
\hline p.D444H & p.K176N & 2 \\
\hline p.D444H & p.T532M & 2 \\
\hline p.D444H & p.I255T & 2 \\
\hline p.D444H & p.N30OH & 2 \\
\hline p.D444H & p.5311fs & 2 \\
\hline p.D444H & p.M86R & 1 \\
\hline p.D444H & p.Y93C & 1 \\
\hline p.D444H & p.T152R & 1 \\
\hline p.D444H & p.R157H & 1 \\
\hline p.D444H & p.N214S & 1 \\
\hline p.D444H & p.L215F & 1 \\
\hline p.D444H & p.D228G & 1 \\
\hline p.D444H & p.T234l & 1 \\
\hline p.D444H & p.C245Y & 1 \\
\hline p.D444H & p.G413s & 1 \\
\hline p.D444H & p.V457M & 1 \\
\hline p.D444H & p.D444H, p.F403V & 1 \\
\hline p.D444H & p.A171T, p.N402S & 1 \\
\hline p.D444H & p/G45R, p.A100T & 1 \\
\hline p.D444H & p.P391S, p.G445V & 1 \\
\hline p.D444H & Targeted & 4 \\
\hline p.D444H & Unknown & 3 \\
\hline p.168V & c. $33 \mathrm{fs}, 44+88 \mathrm{~A}>\mathrm{C}$ (variant) & 1 \\
\hline p.H323R & c. $458 \mathrm{fs}$ & 1 \\
\hline p.R209H & c.310-15delT & 1 \\
\hline
\end{tabular}

only one D444H mutation or were homozygous for the D444H mutation. All three were discharged from the clinic and their biotin was discontinued.

Individuals with partial biotinidase deficiency were routinely started on $5 \mathrm{mg}$ of biotin per day.

Five of these children with partial biotinidase were identified after a younger sibling was identified. Only one of these children had an initial negative newborn screen result and, because the sibling was positive, the testing was repeated and confirmatory testing identified him as also having partial biotinidase deficiency.

One father was identified as having partial biotinidase deficiency after his daughter was diagnosed. He actually had multiple medical issues, including a seizure disorder since age 4 and sensorineural hearing deficits attributed to recurrent otitis media, and he had been in special education classes in school.

Twenty of the children with partial biotinidase deficiency reported a variety of skin problems. These included eczema, thinning scalp hair and flaky scalp, contact dermatitis, folliculitis, peeling of the finger tips, dry skin, itchy knees and elbows, and "bumps" on the arms and legs. Often, these children had their dose increased to $10 \mathrm{mg}$; again, unfortunately, the response to this increase of biotin was not well documented. However, it is likely that the symptoms resolved with or without increases in the biotin dose because these skin issues were not chronic problems on subsequent clinic visits. In addition, there is likely an ascertainment bias in identifying cutaneous findings in children with biotinidase deficiency.

None of the 120 individuals with partial biotinidase deficiency had hypotonia. One had a history of seizures, but this was diagnosed as a neonatal stroke and was not attributed to the biotinidase deficiency. One adolescent with partial biotinidase deficiency was initially treated with biotin; however, for unclear reasons, adequate doses of biotin were discontinued for almost 5 years. The child returned to our clinic with a skin rash and ataxic-like symptoms, which resolved once biotin was reinstated at $5 \mathrm{mg}$ daily and ultimately increased to $10 \mathrm{mg}$ daily. She was subsequently found to have mild sensorineural hearing loss that had not worsened while using biotin. This patient is yet another child with partial deficiency that has developed symptoms of the disorder.

Four other children with partial biotinidase deficiency had hearing problems. Two children had congenital bilateral hearing loss even though they were treated with $10 \mathrm{mg}$ of biotin. The hearing loss in both cases was not likely attributable to the partial biotinidase deficiency because it was present at birth. A third child had borderline bilateral hearing loss and a rash and had his biotin supplementation increased from $5 \mathrm{mg}$ to $10 \mathrm{mg}$, but the effect of the increased dosage of biotin was not available. A fourth child had a 4,000-Hz bilateral sensorineural hearing loss that improved with biotin therapy. Although this child was identified as having the enzyme deficiency on newborn screening, biotin supplementation was not initiated until the child was several years of age, and apparently the hearing loss did not improve with biotin therapy.

Twenty of the individuals reported cutaneous symptoms, including eczema, dry skin, and folliculitis, even while being treated with biotin.

Eleven individuals reported developmental delays. Four had speech and/or language delays of varying degree, one had dyslexia, one had some deficits on a Denver developmental assessment, one had autism diagnosed, one had mild cognitive deficits diagnosed, one was in special education classes at school, and one had to repeat third grade. The mother of the child in special education classes who did not have biotinidase deficiency also has moderate cognitive delays. A genetic evaluation of the child has failed to identify a specific genetic syndrome or cause for their cognitive disability, but it seems unlikely that, given this history, the developmental delay is attributable to the biotinidase deficiency.

\section{DISCUSSION}

Newborn screening for biotinidase deficiency in Michigan over a 25-year period has identified a large number of children with profound biotinidase deficiency and an especially large number of children with partial biotinidase deficiency. The combined 
incidence for profound and partial biotinidase deficiency is higher than that previously reported for worldwide screening. This is mainly attributable to the much higher incidence of partial deficiency in Michigan. Initially, this was thought to be attributable to "over-calling" positive screens, but confirmatory enzymatic analyses and mutation analyses have confirmed the high incidence of partial biotinidase deficiency in the state.

Outcome information is greatly needed for all individuals with the increased number of children identified with various inherited metabolic diseases. Because of the relatively high incidence rate of biotinidase deficiency in Michigan and because essentially all of the children with the disorder are followed by the staff of a single institution, Children's Hospital of Michigan, we were afforded an opportunity to evaluate the outcomes of a fairly large group of individuals with the disorder who are routinely followed in a uniform and consistent manner.

\section{Mutation analysis}

All the mutations identified in individuals with profound biotinidase deficiency have been observed previously, except for an individual who was homozygous for the T234I mutation and one who was compound heterozygous for the novel R209H mutation and a known mutation. ${ }^{11}$

Mutation analysis was performed for many of the children identified with profound and partial biotinidase deficiency, especially because the frequency of partial biotinidase deficiency in our newborn screening population seemed high relative to incidences reported in the literature.

Mutation was used to definitely determine equivocal assignments of children with partial biotinidase deficiency diagnosed, who were heterozygous for profound biotinidase deficiency, or who were homozygous for the $\mathrm{D} 444 \mathrm{H}$ mutation (the most common mutation causing partial deficiency).

Several children with equivocal activity were found to have biotinidase activity in the partially deficient/carrier range and were subsequently shown to be homozygous for the $\mathrm{D} 444 \mathrm{H}$ mutation. These children were subsequently found to have activities most consistent with that of heterozygotes for profound biotinidase deficiency; therefore, biotin therapy was not continued.

Previous reports indicate that $\sim 98 \%$ of the individuals with partial biotinidase deficiency have the $\mathrm{D} 444 \mathrm{H}$ mutation as one of their alleles in combination with an allele for profound biotinidase deficiency. ${ }^{13}$ We have observed that $97 \%$ of our individuals with partial biotinidase deficiency have at least one of their alleles as the $\mathrm{D} 444 \mathrm{H}$ mutation. In addition, the majority of the second alleles for profound biotinidase deficiency have been observed in other newborn screening population. ${ }^{14}$ However, we did observe several individuals who had a novel second mutation $^{11}$ that, based on the enzymatic activity, is expected to cause profound biotinidase deficiency.

\section{Clinical findings}

In our group of children with profound biotinidase deficiency, none experienced development of major neurological or cutaneous problems while routinely taking biotin.
In general, anecdotal experience indicates that individuals with partial biotinidase deficiency rarely exhibit symptoms associated with untreated profound biotinidase deficiency unless the child is stressed by an infection or gastroenteritis and/ or is not taking the biotin for an extended period of time. ${ }^{15,16}$

We did find two interesting and informative symptomatic individuals with partial biotinidase deficiency in our population. One was an adolescent who exhibited clinical symptoms when therapy was discontinued for a short period of time. The second was a symptomatic adult who was first identified as having partial biotinidase deficiency only after his daughter was identified as having the disorder. These cases further support that individuals with partial deficiency can develop symptoms.

Sixteen percent of those with partial biotinidase deficiency reported skin findings, including eczema, dry skin, hair texture, and distribution issues, and folliculitis. Atopic dermatitis or eczema has also been a concern, but is known to affect between $10 \%$ and $20 \%$ of children worldwide. ${ }^{17}$ The best way to determine whether the skin abnormality is attributable to the biotinidase deficiency is to determine whether the skin finding resolves after increasing the dose of biotin. In essentially all cases, biotin alone did not resolve the problem. Therefore, the skin problem, particularly eczema, was not likely attributable to the biotinidase deficiency.

With the exception of a single child who had a seizure during the neonatal period, which was determined to be caused by a stroke, none of the other children with profound or partial biotinidase deficiency reported episodes of lethargy, seizures, or ataxia.

The vision problems reported for this population were usually common findings, ${ }^{18}$ such as myopia warranting glasses or contact lenses; one child had a congenital cataract, but this was likely unrelated to biotinidase deficiency. None of the children was reported to have optic atrophy.

Six children (4.8\%), all with partial biotinidase deficiency, had documented hearing loss. One child had congenital hearing loss, two had bilateral sensorineural hearing loss, and three had decreased hearing or sensorineural hearing loss. One of these two children was found to have been receiving inadequate doses of biotin for several years. Overall, hearing loss occurs in 1 to 2 in 1,000 newborns and in 2 in 1,000 young children. The prevalence of sensorineural hearing loss in our population was higher than that of the general population. ${ }^{19}$

A major concern of several of the parents was their child's developmental progress, specifically, their grades in class. One child repeated a grade and another child required special education. One child with partial biotinidase deficiency was diagnosed with autism. Again, it is expected that a small number of any population will exhibit these issues. ${ }^{20}$ In some cases, increasing the biotin dosage, if attempted, did not ameliorate the problem.

Approximately 3\% of the children with partial biotinidase deficiency had speech and/or language delays. Approximately $10 \%$ to $15 \%$ of 2 -year-old children in the general population have language delay, and $4 \%$ to $5 \%$ have language delay after 3 years. $^{21}$ Because speech and language problems are 
also common in the general population, we cannot be certain whether the speech/language issues were caused by the partial biotinidase deficiency.

There have been few studies, such as ours, that have followed these children for extended periods of time..$^{4,22-24}$ Review of the individuals with profound biotinidase deficiency appears to support that if these children are identified early within the newborn period, started on biotin therapy, and have good compliance, then they can have normal physical and cognitive development. There was low frequency of medical problems associated with individuals with the deficiency who became symptomatic before diagnosis and treatment.

\section{Conclusions}

There seems to be a skewing of both profound and partial biotinidase deficiency toward children of younger ages. The most likely reasons for this are that older individuals with either profound or partial biotinidase deficiency have remained asymptomatic while on biotin, which is readily available as an over-the-counter nutrient, or they have moved out of the region of the clinics in Michigan. These issues obviously show the disadvantages of retrospective studies in which some of the specific data are lacking in the medical records used in this study, such as whether the skin issues reported respond to increases in biotin therapy. Perhaps long-term prospective studies will better answer these specific questions, but there is still the likely loss of participants with biotinidase deficiency because this disorder is so readily treatable and it does not need the scrutiny that is required for many of the other inherited metabolic disorders.

Because the clinical symptoms that usually develop in untreated individuals with profound biotinidase deficiency are often general, common, and potentially attributable to other etiologies, it is often difficult to determine if an enzyme-deficient individual's clinical features are attributable to biotinidase deficiency. In most instances, other causes were more likely, especially because compliance of biotin therapy did not seem to be an issue. Compliance is usually confirmed by the individuals admitting that they have not been taking their biotin or that the symptoms resolve when biotin is reinstituted..$^{15}$

Biotin therapy appears to prevent development of neurologi$\mathrm{cal}$ and cutaneous problems in our population with biotinidase deficiency. In fact, if a child does develop any of these associated problems, then compliance with biotin therapy should be the first consideration, and increasing the dose of the biotin, especially after puberty, might need to be attempted. However, after compliance or dosage issues have been excluded, other causes of these clinical issues must be addressed as if the child did not have the enzymatic disorder. In addition, the Michigan experience further supports the benefit of newborn screening for biotinidase deficiency.

\section{ACKNOWLEDGMENT}

This work was funded in part by the Safra Research Fund at Henry Ford Hospital (B.W.).

\section{DISCLOSURE}

G.L.F. is the medical director for laboratories that offer biotinidase enzymatic and molecular testing and the president-elect of the ACMG. The other authors declare no conflict of interest.

\section{REFERENCES}

1. Pispa J. Animal biotinidase. Ann Med Exp Biol Fenn 1965;43(suppl 5):1-39

2. Wolf B, Feldman GL. The biotin-dependent carboxylase deficiencies. Am J Hum Genet 1982;34:699-716.

3. Wolf B, Grier RE, Allen RJ, Goodman SI, Kien CL. Biotinidase deficiency: the enzymatic defect in late-onset multiple carboxylase deficiency. Clin Chim Acta 1983;131:273-281.

4. Wolf B. Biotinidase deficiency: "if you have to have an inherited metabolic disease, this is the one to have". Genet Med 2012;14:565-575.

5. Wolf B. Disorders of biotin metabolism. In: Scriver CR, Beaudet AL, Sly WS, Valle D (eds). The Metabolic and Molecular Bases of Inherited Disease, 8th edn. New York: McGraw-Hill; 2001;3935-3962.

6. Wolf $B$, Heard GS, Jefferson LG, et al. Neonatal screening for biotinidase deficiency: an update. J Inherited Metab Dis 1986;9(suppl 2):303-306.

7. Wolf $B$, Heard GS. Screening for biotinidase deficiency in newborns: worldwide experience. Pediatrics 1990;85:512-517.

8. Wolf B, Heard GS, Jefferson LG, Proud VK, Nance WE, Weissbecker KA. Clinical findings in four children with biotinidase deficiency detected through a statewide neonatal screening program. N Eng/ J Med 1985;313:16-19.

9. Heard GS, Secor McVoy JR, Wolf B. A screening method for biotinidase deficiency in newborns. Clin Chem 1984;30:125-127

10. Pomponio RJ, Hymes J, Reynolds TR, et al. Mutations in the human biotinidase gene that cause profound biotinidase deficiency in symptomatic children: molecular, biochemical, and clinical analysis. Pediatr Res 1997;42:840-848.

11. Li H, Spencer $L$, Nahhas F, et al. Novel mutations causing biotinidase deficiency in individuals identified by newborn screening in Michigan including an unique intronic mutation that alters mRNA expression of the biotinidase gene. $\mathrm{Mol}$ Genet Metab 2014;112:242-246.

12. Wolf $B$. Worldwide survey of neonatal screening for biotinidase deficiency. J Inherit Metab Dis 1991;14:923-927

13. Swango KL, Demirkol M, Hüner $G$, et al. Partial biotinidase deficiency is usually due to the $\mathrm{D} 444 \mathrm{H}$ mutation in the biotinidase gene. Hum Genet 1998;102:571-575.

14. Norrgard KJ, Pomponio RJ, Hymes J, Wolf B. Mutations causing profound biotinidase deficiency in children ascertained by newborn screening in the United States occur at different frequencies than in symptomatic children. Pediatr Res 1999;46:20-27.

15. Wolf $\mathrm{B}$. Clinical issues and frequent questions about biotinidase deficiency. Mol Genet Metab 2010;100:6-13.

16. Wolf B. Biotinidase deficiency. In: Pagon RA, Bird TD, Dolan CR, Stephens K, Adam MP (eds). GeneReviews (Internet). Seattle, WA: University of Washington, 2012. www.ncbi.nih.gov/books/NBK1322.

17. Flohr C, Mann J. New insights into the ophthalmology of childhood atopic dermatitis. Allergy 2013;69:3-16.

18. Vitale $S$, Sperduto RD, Ferris FL 3rd. Increased prevalence of myopia in the United States between 1971-1972 and 1999-2004. Arch Ophthalmol 2009;127:1632-1639.

19. American Speech-Language-Hearing Association. The prevalence and incidence of hearing loss in children. www.asha.org/public/hearing/Prevalence-andIncidence-of-Hearing-Loss-in-Children/.

20. Center for Disease Control and Prevention. Data and Statistics, Autism Spectrum Disorder. www.cdc.gov/ncbddd/autism/data.html.

21. Nelson HD, Nygren P, Walker M, Panoscha R. Screening for speech and language delay in preschool children: systematic evidence review for the US Preventive Services Task Force. Pediatrics 2006;117:e298-e319.

22. Möslinger D, Stöckler-Ipsiroglu S, Scheibenreiter $S$, et al. Clinical and neuropsychological outcome in 33 patients with biotinidase deficiency ascertained by nationwide newborn screening and family studies in Austria. Eur J Pediatr 2001;160:277-282

23. Moslinger D, Stockler-Ipsiroglu S, Scheibenreiter $\mathbf{S}$, et al. Clinical and neuropsychological outcome in 33 patients with biotinidase deficiency ascertained by nationwide newborn screening and family studies in Austria. Eur J Pediatr 2002;161:167-168.

24. Möslinger D, Mühl A, Suormala T, Baumgartner R, Stöckler-lpsiroglu S. Molecular characterisation and neuropsychological outcome of 21 patients with profound biotinidase deficiency detected by newborn screening and family studies. Eur J Pediatr 2003;162(suppl 1):S46-\$49. 